\title{
MDI-SOHO Measures of Solar Radius Variation
}

\author{
M. Emilio ${ }^{1}$, J. R. Kuhn \\ Institute for Astronomy, University of Hawaii, Honolulu HI 96822 \\ R. I. Bush and P. Scherrer \\ Stanford University, Stanford, CA 94305
}

\begin{abstract}
Is the Solar Radius a constant? Ground experiments show evidence of possible variations in the visual solar semi diameter that are correlated with the solar activity. Those measurements are limited by the Earth's atmospheric turbulance. It is importance to accurately determine the solar radius variations because of their implication for stellar structure and possible relation to the terrestrial climate. Here we report on data from a space experiment (MDI-SOHO) used to detect solar diameter fluctuations. We stabilish a superior limit for changes in the solar radius.
\end{abstract}

\section{Introduction}

Experiments during the last century, and performed with different techniques, show evidence of significant variations in the visual solar diameter (Gilliland 1980; Ribes it et al. 1991; Laclare 1996). The classical methods for solar radius measurement are based on timing meridian transit of the borders of the Sun, on observations of the limits of totality of solar eclipses, and on transits of Mercury. More recently the prismatic astrolabe technique uses the timing of two images of the same Sun (on direct and another reflective), defined by a fixed almulcantar (Leister \& Benevides-Soares 1990; Laclare et al. 1996). The objective has been to obtain high quality mearurements of the solar radius because of their possible implications for the Earth's climate. Precise measurements of solar radius variations can also test and improve our understanding of the Sun.

Solar energy is produced constantly by nuclear fusion in the core. Photon's created in the core reach the photosphere and are radiated after nearly a million years. Thus, given the observed irradiance variability at the surface of $0.1 \%$ (Wilson \& Hudson 1998) and similarly in the luminosity (Kuhn, Libbrecht, \& Dicke 1988), there must be an intermediate energy reservoir somewhere between the core and the photosphere. Several mechanisms exist for storing energy during a solar cycle (e.g. gravitational or magnetic fields) and each leads to distinct perturbations in the equilibrium stellar structure and changes in the diameter.

\footnotetext{
${ }^{1}$ Instituto Astronômico e Geofísico-Universidade de São Paulo CP 3386 São Paulo-SP 01060-970 BRAZIL
} 
The magnitude of the radius fluctuation, compared to the irradiance change, during a solar cycle contains information on where and how energy is stored.

We take $W=\frac{\delta r / r}{\delta I / I}$ to describe the ratio of radius and irradiance changes over a solar cycle. Various models predict a wide range of values for W. For example, depending on the details of the solar model and the form of the perturbation, theoretical estimates of $\mathrm{W}$ range between $2 \times 10^{-4}$ (Spruit 1982), $8 \times 10^{-4}$ (Gilliland 1980), $5 \times 10^{-3}$ (Dearborn \& Blake 1980) and $7.5 \times 10^{-2}$ (Sofia \& Endal 1979). Given the solar cycle irradiance amplitude of $0.1 \%$ a radius sensitivity of at least 70 milliarcsec is needed to observationally discriminate between some of these models.

Given the small magnitude of the diameter change signal in the groundbased measurements, it is clear that solar radius observations are limited by "seeing" affects in the Earth's atmosphere. The Michelson Doppler Imager (MDI) instrument (Scherrer et al. 1995), which is one component of the Solar and Heliospheric Observatory (SOHO) satellite, offers the promise of very accurate solar radius measurements which can only be obtained from space.

\section{Limb Observations}

The dataset consists of a 6 pixel wide annulus of intensity measurements obtained from solar images produced during the routine operation of the MDI experiment. The 1 minute cadence images are low-pass filtered (to remove solar 5 -minute p mode intensity oscillations) and the limb pixels (2" / pixel) are downlinked every 12 minutes. These data were obtained between April 19, 1996 and June 24 1998. The limb position $\delta r\left(\theta_{i}\right)$, from each bin is obtained by finding the inflection point in the limb darkening functions. This is determined by fitting $d I\left(r, \theta_{i}\right) / d r$ to a Gaussian plus quadratic background. The zero of this function is taken to be the limb position.

The Figure 1 shows the daily average residual of the semi diameter after subtract the effect caused by the MDI front window temperature and focus change (Emilio et al. 2000). The radius fluctuation measurements, $\delta r^{\prime}(t)$, define an upper limit on possible secular variations. The residual linear variation in the Sun's radius using the data in Figure 1 is $8.1 \pm 0.9$ milliarcsec/year. We have also searched for evidence of a solar cycle variation by fitting a smoothed sunspot function $\mathrm{S}(\mathrm{t})$ to $\delta r^{\prime}(t)$.

We use a two-parameter linear model of the form $\alpha+\beta S(t)$ to fit for a solar cycle variation in the radius change dataset. We find that the MDI data imply $\beta_{M D I}=0.3 \pm 0.03$. These coefficients have units of milliarcsec per sunspot number. From $\beta_{M D I}$ and the peak-to-valley excursion of the sunspot function, $\delta S$, one would conclude that the solar cycle radius variation is $\delta r_{c y c l e}=$ $\delta S \beta_{M D I}=+21 \pm 3$ milliarcsec. In fact, because we cannot rule out the existence of unmodeled slow temperature or MDI instrument changes, we believe that $\delta r_{c y c l e}$ represents an upper limit to the magnitude of a real cyclic solar size variability. Thus we take $2 \times 10^{-2}$ as the upper limit of $|W|$. 


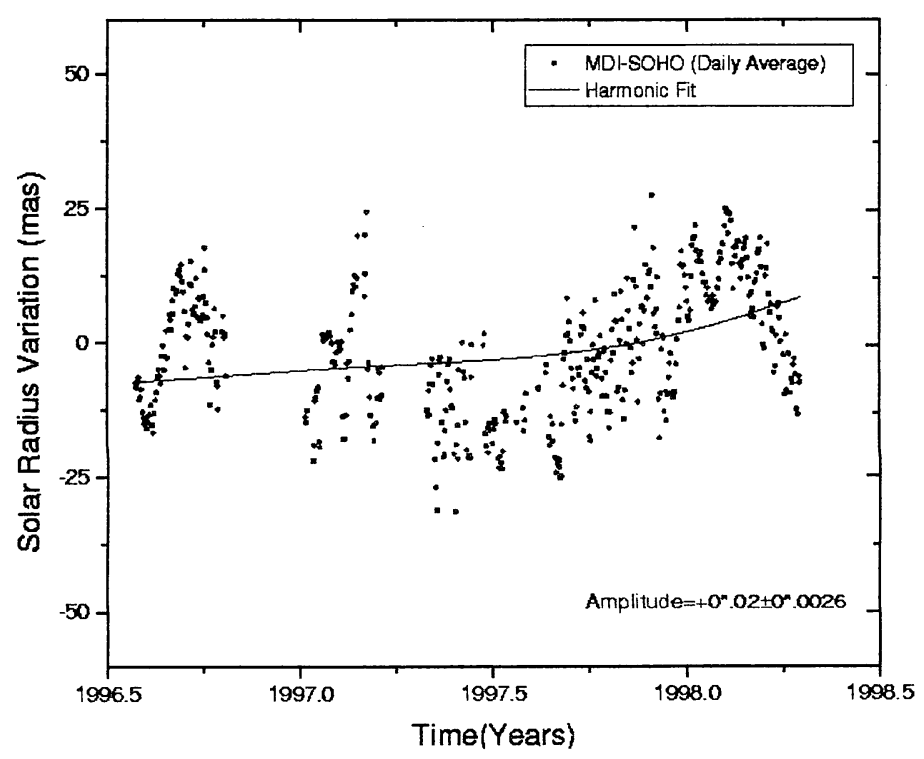

Figure 1. This figure shows daily average solar radius variations after removing the MDI front window temperature contribution. The residual linear variation in the Sun's radius is $8.1 \pm 0.9$ milliarcsec/year. The smooth curve in this figure shows a fit to the smoothed sunspot number during this period.

\section{Discussion}

Sofia \& Endal (1979) argued that solar cycle changes which affect the convective efficiency near the photosphere will have a comparatively large affect on the solar radius. Their calculated value of $\mathrm{W} \approx 0.075$ is ruled out by these measurements, suggesting that solar cycle irradiance changes are not caused by such superficial fluctuations in the outer (superadiabatic) layers of the sun. It is possible to improve the radius detection sensitivity by more than one order of magnitude, either with a longer measurement time baseline from MDI, or with other dedicated satellite observations. In either case, it is clear that precise solar radius observations have reached a level of accuracy that particular models of solar cycle variability may be directly addressed. These new astrometric results support the view that changes deep in the solar interior are required to account for the observed irradiance variability.

\section{References}

Dearborn, D. S. P., \& Blake, J. B. 1980, ApJ, 237, 616

Emilio, M., Kuhn J. R., Bush R. I., \& Scherrer, P. 2000 ApJ, in press 
Gilliland, R. L. 1980, ApJ, 248, 1144

Kuhn, J. R., Libbrecht, K. G., \& Dicke, R. H. 1988, Science, 242, 908

Leister N. V., Benevides-Soares P. 1990, C. R. Acad. Sci. Paris, t. 311, s. II, 399

Laclare, F., Delmas, C., Coin, J. P., \& Irbah A. 1996, Solar Phys., 166, 211

Parkinson, J. H., Morrison, L. V., \& Stephenson, F. R. 1980, Nature 288, 548

Ribes, E., Beardsley, B., Brown, T. M., Delache., Ph., Laclare, F., \& Leister, N. V. 1991, in The Sun in Time, ed. C. P. Sonett, M. S. Giampapa, \& M. S. Matthews (Arizona Univ. Press), 59

Scherrer, P. H. et al. 1995, Solar Phys., 162, 129

Sofia, S., \& Endal, A. S. 1979, Science, 204, 1306

Spruit, H. C. 1982 A\&A, 108, 348

Wilson, R. C., \& Hudson, H. S. 1988, Nature, 332, 810 